\title{
Correction to: Expanding Diversity and Common Goal of Regulatory T and B Cells. I: Origin, Phenotype, Mechanisms
}

\author{
Katarzyna Bocian ${ }^{1}$ - Ewelina Kiernozek ${ }^{1}$ Joanna Domagała-Kulawik ${ }^{2}$. \\ Grażyna Korczak-Kowalska ${ }^{1,4}$ - Anna Stelmaszczyk-Emmel ${ }^{3} \cdot$ Nadzieja Drela $^{1}$
}

Published online: 10 October 2017

(C) L. Hirszfeld Institute of Immunology and Experimental Therapy, Wroclaw, Poland 2017

\section{Erratum to: Arch. Immunol. Ther. Exp.}

DOI 10.1007/s00005-017-0469-3

The original article has been published without acknowledgment section. The acknowledgement section is given below for your reading.

Acknowledgements This work was supported by Grant no. STRATAGMED2/267976/13/NCBR/2015.

The online version of the original article can be found under doi:10.1007/s00005-017-0469-3.

Katarzyna Bocian

kbocian@biol.uw.edu.pl; katarzyna@bocian.waw.pl

$\triangle$ Ewelina Kiernozek

ekiernozek@biol.uw.edu.pl

1 Department of Immunology, Faculty of Biology, University of Warsaw, Warsaw, Poland

2 Department Pneumonology, Medical University of Warsaw, Warsaw, Poland

3 Department of Laboratory Diagnostics and Clinical Immunology of Developmental Age, Medical University of Warsaw, Warsaw, Poland

4 Department of Clinical Immunology, Transplantation Institute, Medical University of Warsaw, Warsaw, Poland 